\title{
FPGA-based Weblab Infrastructures Guidelines and a prototype implementation example
}

\author{
Ricardo J. Costa ${ }^{1}$, Gustavo R. Alves ${ }^{1}$, Mário Zenha-Rela ${ }^{2}$, Rob Poley ${ }^{3}$, Campbell Wishart ${ }^{3}$ \\ ISEP/CIETI/LABORIS ${ }^{1}$, FCTUC/CISUC ${ }^{2}$, Heriot-Watt University/DSG ${ }^{3}$ \\ rjc@isep.ipp.pt; gca@isep.ipp.pt; mzrela@dei.uc.pt; rjp@macs.hw.ac.uk; campbell@wishart.org.uk
}

\begin{abstract}
Recent trends show an increasing number of weblabs, implemented at universities and schools, supporting practical training in technical courses and providing the ability to remotely conduct experiments. However, their implementation is typically based on individual architectures, unable of being reconfigured with different instruments/modules usually required by every experiment. In this paper, we discuss practical guidelines for implementing reconfigurable weblabs that support both local and remote control interfaces. The underlying infrastructure is based on reconfigurable, low-cost, FPGA-based boards supporting several peripherals that are used for the local interface. The remote interface is powered by a module capable of communicating with an Ethernet based network and that can either correspond to an internal core of the FPGA or an external device. These two approaches are discussed in the paper, followed by a practical implementation example.
\end{abstract}

Keywords: Weblabs, FPGAs, reconfigurable systems, remote labs, remote experimentation.

\section{INTRODUCTION}

Weblabs are becoming a widely used resource for supporting technical courses, allowing students to interact with real equipment from everywhere and at anytime without physically being present in a classical lab. This new type of labs are an added value for education, enabling to include more practical work and giving students the ability to repeat the experiments done in classical labs. Among others, two aspects have been contributing to increase the number of weblabs implemented at universities and schools, namely the technological evolution that causes several instruments to be factory-equipped with Ethernet physical interfaces, and the increasing number of students in technical courses, requiring more labs to be available for their practical training, which may pose economical constraints for institutions. Commonly less expensive than a classical lab, which requires several workbenches for students training, the implementation of a weblab infrastructure may also became expensive depending on the cost of the adopted equipment. Besides increasing flexibility and costs savings, other advantages should also be pointed to weblabs, namely the contribution they bring for increasing collaboration among institutions by enabling knowledge sharing in specific areas. All these aspects contribute to the number and variety of weblabs available today, although a large majority is focused in engineering courses $[1,2,3,4]$.

Typically each weblab infrastructure is developed following specific and distinct technical implementations, with several hardware and software architectures that use different programming languages to remotely control the equipment [5,6,7]. These aspects are impairing a higher proliferation of weblabs in different areas, while the difficulties of reusing and interfacing different modules used in their infrastructures are decreasing the collaboration among institutions. To overcome these problems, some authors have being proposing generic software and hardware architectures $[8,9]$, but more efforts and contributions are needed. We contribute to this endeavor by proposing a reconfigurable infrastructure, focused at the hardware level, which allows creating, sharing and reusing instruments and other experiment-related modules.

Section II discusses a set of guidelines on how to create reconfigurable weblabs infrastructures capable of reusing different instruments and modules. Section III presents two approaches that allow remotely controlling and monitoring those infrastructures and describes an architecture for sharing instruments and modules typically used in weblabs. Section IV describes an implementation of an FPGA-based weblab infrastructure, and section $\mathrm{V}$ presents the web interface developed for controlling the implemented infrastructure. Section VI concludes the paper.

\section{HARDWARE SPECIFICATION GUIDELINES}

To increase weblabs acceptance, two aspects must be considered regarding their implementation:

- costs are minimized;

- a flexible reconfigurable architecture is adopted.

Nowadays, weblabs include PCs with several individual instruments connected through instrumentation buses like PXI (PCI eXtended to Instrumentation), GPIB, and more recently with LXI (LAN eXtensions for Instrumentation). Although these architectures guarantee high performance by using dedicated instruments, they are expensive and require several hardware/software modules with specific functions eventually not necessary for some experiments. To reduce expenses and to target a universal architecture for implementing weblab infrastructures with reconfiguration capabilities, two devices must be considered: $\mu \mathrm{C} / \mu \mathrm{Ps}$ 